\title{
Reaction time for horizontal versus vertical line-length discrimination
}

\author{
ROBERT GOTTSDANKER and JEROME D. TIETZ \\ University of California, Santa Barbara, California
}

\begin{abstract}
In an experiment with 8 men and women, a small but statistically significant effect on reaction time (RT) was found with respect to horizontal versus vertical line-length discrimination. This effect was in the form of a crossing interaction between axis of orientation and the short-long ratio of line lengths. With a relatively high value of this ratio (the more difficult discrimination), horizontal choice was faster; with a lower value, vertical choice was faster. An interpretation of the crossing interaction is that two opposing factors were involved: greater horizontal than vertical sensitivity and greater left-right than up-down ambivalence. It is recommended that in RT experiments comparing parallel and orthogonal stimulus-response mapping, all four combinations of horizontal and vertical stimulus and response axes be employed.
\end{abstract}

A study comparing reaction time (RT) of line-length discrimination for horizontal and vertical stimulus orientation in the frontal plane is of interest for two reasons. First, it tests the validity of certain uses of the variable of parallel versus orthogonal stimulus-response (S-R) mapping in typical RT experiments. Second, a methodological and substantive contribution is made to the literature on meridional anisotropy.

The term "S-R compatibility" has acquired a number of divergent meanings. As Sanders (1980, p. 339) points out, such differences make interstudy comparisons very difficult-for example, in integrating the findings in which the additive-factors method (Sternberg, 1969) has been employed. For a considerable range of problems, the variable of parallel versus orthogonal S-R mapping could be used instead. When two response units (hands or fingers) are horizontally displaced, a comparison of horizontal line lengths would constitute the parallel mapping and a vertical comparison would constitute the orthogonal mapping. This variable would be expected to affect translation time without introducing response competition, as with reversed coding. Also, the "noncompatible" discrimination required is preferable to an arbitrary discrimination (e.g., between colors), since the stimulus is of the same kind as the "compatible" discrimination. Finally, difficulty of discrimination may be employed as an additional variable. The procedure with two horizontally displaced response units that has been outlined relies on the assumption that horizontal and vertical discriminations are made equally rapidly. Otherwise, the variable of parallel-orthogonal mapping would be confounded with discriminability. This is the assumption tested in the present experiment.

This research was supported by Grant 2 RO1 MH39359 to the University of California from the National Institute of Mental Health. Requests for reprints should be sent to Robert Gottsdanker, Department of Psychology, Santa Barbara, California 93106-9660 (e-mail: bgottsda@ucsbuxa.bitnet).
A study of this kind would also contribute to our knowledge of meridional anisotropy. Although considerable evidence exists to support the claim that acuity is poorer on oblique stimulus orientations than on horizontal or vertical orientations (e.g., Leibowitz, 1955; Regan \& Price, 1986), there have been fewer comparisons of horizontal versus vertical acuity. The threshold results that have been obtained have not, in general, supported the existence of horizontal-vertical anisotropy of acuity. Leibowitz (1953) found no difference in acuity between horizontal and vertical sine-wave gratings. Leibowitz (1955) also found no consistent horizontal-vertical difference for vernier acuity, and McKee and Westheimer (1978) found, in addition, that with sufficient practice, an oblique orientation was not at a disadvantage. For orientation discrimination, Sulzer and Zener (1953) did report a lower single-line threshold for horizontal than for vertical direction, indicative of finer vertical displacement acuity. However, more recent work has not supported this finding. Heeley and Timney (1988) found at least as small a threshold for the vertical orientation, both for thin single lines and sine-wave gratings. Regan and Price (1986) found no consistent horizontalvertical difference in discrimination of orientation of sinewave gratings. Still, line-length discrimination per se apparently has not been compared for the horizontal and vertical meridians. Moreover, RT methods do not appear to have been employed previously in the comparison of horizontal and vertical distance differentiation to provide information on suprathreshold sensitivity.

In the present study, by using a contingent (or $\mathrm{c}$-) reaction, the issue of S-R compatibility is circumvented in determining whether there is more rapid discrimination along one of the two meridians. Furthermore, by using two different levels of difficulty (short-long ratios of line lengths), a second test of discriminability is possible. With meridional anisotropy of sensitivity, in comparing line lengths there should be a more than additive interaction between orientation and short-long ratio (Sternberg, 1969). 


\section{METHOD}

\section{Subjects}

The subjects, who were paid participants, were 8 (6 men and 2 women) right-handed graduate students at the University of California, Santa Barbara. Ages ranged from 24 to 41 years.

\section{Task}

Each subject was seated in a dimly illuminated, sound-shielded room in front of an amber CRT (Samsung MD-12526) display at an eye distance of about $70 \mathrm{~cm}$. On each trial, the subject was presented with a stimulus line divided into two segments of unequal length by a short perpendicular line. The subject was instructed to press the response button only on those trials on which the shorter segment was in the designated direction, which randomly occurred on half the trials. On different blocks of trials, the orientation of the stimulus line was either horizontal or vertical.

The transit-signal method (Gottsdanker, 1970) was employed to reduce temporal uncertainty with respect to stimulus onset on a trial. As the meridional differences could not be expected to be very great in light of previous studies, the consequent reduction in variance provided by this technique could be useful. Each trial began with the appearance of a timing dot and a short dividing line, which was vertical for the horizontal orientation of the forthcoming stimulus line and vice versa. In either orientation, the dividing line was located at the center of the display. For the horizontal orientation, the starting point of the timing dot was near the bottom edge of the display. For the vertical orientation, the timing dot initially appeared near the left edge of the display. In either case, the timing dot moved at a constant velocity toward the dividing line, with which it merged after $3 \mathrm{sec}$. At this instant, time zero, the stimulus line appeared across the dividing line, with the subject instructed to press the response button if the shorter segment was in the designated direction, which randomly occurred on half the trials. On the display, the subjects were provided feodback regarding reaction times and errors after every response. The stimulus events for the horizontal orientation are represented in Figure 1 at $-3 \mathrm{sec},-1 \mathrm{sec}$, and time zero.

Within each orientation (horizontal or vertical), there were two levels of line-segment similarity, with the shorter segment of the stimulus line being either $5 / 6$ (providing the more difficult discrimination) or $1 / 2$ the length of the longer segment. For each of the ratios, there were also two different total line lengths: $4.2 \mathrm{~cm}$ (Task 1) and $5.1 \mathrm{~cm}$ (Task 2) for the $5 / 6$ ratio, and $2.1 \mathrm{~cm}$ (Task 3 ) and $4.2 \mathrm{~cm}$ (Task 4) for the $1 / 2$ ratio. With the different line lengths for the ratio, the subject was prevented from basing the response on a particular end location of the stimulus line and was required to make a true length comparison. Finally, there were two sets of instructions. In one instruction for the horizontal orientation, the subject was required to respond if the shorter segment was

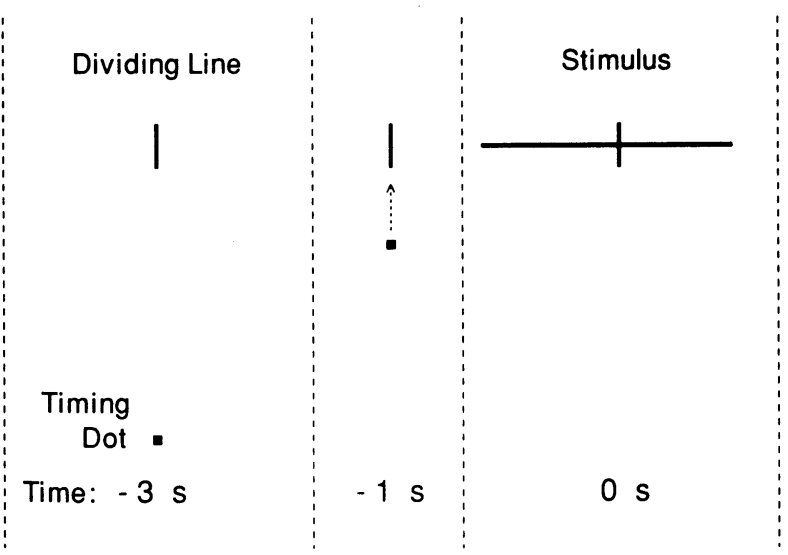

Figure 1. The stimulus events during a trial, shown for the horizontal orientation and 5/6 line-length ratio. The timing dot, shown enlarged, moved with a constant velocity toward the dividing line from -3 sec to 0 sec (time zero).

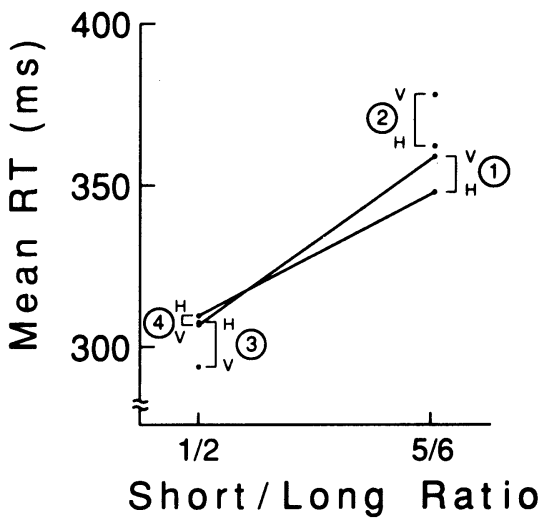

Figure 2. Overall mean RTs for the horizontal and vertical orientations on the four numbered tasks. Tasks 1 and 4, connected by lines, had the same total line length, $4.2 \mathrm{~cm}$. The total line lengths for Tasks 2 and 3 were 5.1 and $2.1 \mathrm{~cm}$, respectively.

to the left of the crossing line; in the other instruction, a response was required if the shorter segment was to the right. Comparable instructions for the vertical orientation concerned the top and bottom segments.

Both the stimulus and crossing lines were $1.00 \mathrm{~mm}$ wide when oriented horizontally and $0.75 \mathrm{~mm}$ wide when oriented vertically. The consequences of this inequality, which was due to resolution limitations in the graphical display, are considered in the discussion. The timing dot was $1.00 \mathrm{~mm}$ high and $0.75 \mathrm{~mm}$ wide. The luminance of each of the lines and the timing dot was $2.8 \mathrm{log}$ units above focal threshold under the average adaption conditions of the experiment. A force of about $0.2 \mathrm{~N}$ through a distance of $\mathbf{2} \mathrm{mm}$ was sufficient to operate the microswitch to which the response button was attached. All programming of the display, measurement and recording of RTs (to the nearest millisecond) and errors, and the providing of feedback were controlled by an IBMcompatible Standard 286/10 computer.

\section{Procedure}

Each subject was tested in eight 45-min sessions, each conducted on a different day. A session consisted of 320 trials, with a break every 80 trials. Within a session, one of the two orientations, one of the two short-long ratios, and one of the two instructions were employed throughout. These 8 subconditions were administered to the 8 subjects in a balanced-square design.

\section{RESULTS}

Data analysis was based on the last 101 trials. Overall, mean RT on correct trials was almost identical for the two orientations: 332 and $334 \mathrm{msec}$ for horizontal and vertical, respectively. However, for the $5 / 6$ line-length ratio, mean vertical RT was longer ( 359 vs. $348 \mathrm{msec}$ for Task 1 and 378 vs. $362 \mathrm{msec}$ for Task 2), whereas for the $1 / 2$ ratio, mean vertical RT was at least slightly shorter (294 vs. $307 \mathrm{msec}$ for Task 3 and 307 vs. $310 \mathrm{msec}$ for Task 4). The relevant comparisons are shown in Figure 2.

A repeated measures analysis of variance (ANOVA) was conducted on mean RTs, with the variable of instruction nested within the variable of orientation. The main effect of orientation, obviously, was not significant $(F<1)$, but the interaction between task and orientation was significant $\left[F(3,21)=4.71, M S_{\mathrm{e}}=304.84, p<.02\right]$. Differences among tasks were significant $[F(3,21)=31.78$, $\left.M S_{\mathrm{e}}=1162.67, p<.01\right]$. In the Scheffé comparisons, 
RT differences between tasks having the different shortlong ratios were significant $(p<.01)$, but differences within the same ratio were not significant.

Overall, there was a slightly higher proportion of errors for the vertical orientation $(0.20$ vs. 0.17$)$. The same kind of ANOVA that was applied to the RT data was conducted on the proportion of errors, that is, responses made on trials calling for no response. The main effect of orientation reached borderline significance $\left[F(1,7)=5.48, M S_{\text {e }}\right.$ $=48.78, p=.0517]$. The main effect of task was significant $\left[F(3,21)=10.44, M S_{\mathrm{e}}=142.09, p<.01\right]$. In one relevant Scheffé comparison, in the two cases in which total line length was the same $(4.2 \mathrm{~cm})$, there were significantly more errors for Task 1 , with the $5 / 6$ ratio, than for Task 4 , with the $1 / 2$ ratio $(p<.01)$. When the ratio was the same (1/2), there were significantly more errors for Task 3, with a line length of $2.1 \mathrm{~cm}$, than for Task 4 , with a line length of $4.2 \mathrm{~cm}(p<.01)$. Thus, the proportion of errors was greater with more similar line segments and with a shorter total line length. Finally, there was a significant interaction between task and instruction. Inexplicably, on Task 4 only, errors were reduced appreciably by instructing the subject to respond if the shorter segment was to the left or to the top, depending on orientation, rather than the reverse $\left[F(6,42)=3.17, M S_{\mathrm{e}}=\right.$ $46.52, p<.02$ ].

\section{DISCUSSION}

It will be recalled that meridional anisotropy in line-length discrimination was expected to be revealed by a shorter mean RT for one of the two orientations, coupled with a more than additive interaction between line-length ratio and orientation. What was found instead was a crossing interaction, with a horizontal advantage on the more difficult discrimination and a vertical advantage on the easier discrimination. An interaction between orientation and line-length ratio, regardless of its form, indicates that the two variables affect some processing stage, or stages, in common (Sternberg, 1969). Thus, the effect of compatibility would be overestimated in an experiment using the two hands with a horizontal discrimination for the compatible arrangement and a vertical discrimination for the noncompatible arrangement. The confounding could be eliminated by using a single response lever that could be moved either left and right or up and down, each direction used with both a horizontal and a vertical display.

A possible explanation for a crossing interaction between orientation and line-length ratio is that there were two opposing factors: greater horizontal than vertical sensitivity and greater left-right than up-down confusability or ambivalence (Corballis \& Beale, 1983). The latter factor had not been considered in the design of the experiment. Since the analysis for each subcondition was based on the last 101 of the 320 trials, the present findings would not appear attributable to lack of practice (see McKee \& Westheimer, 1978). It has been shown (Farrell, 1979) that left-right judgments of position have a longer RT than up-down judgments. In those studies, the discriminative aspect was very easy. It could be argued that with the present $5 / 6$ ratio, the sensitivity advantage for the horizontal axis outweighed the left-right ambivalence dis- advantage, whereas with the $1 / 2$ ratio, the ambivalence disadvantage was greater. Here, it may be noted that RT has been found to be shorter for naming the one dashed side of a geometric figure when it was up or down than when it was left or right (Greene, Plastow, \& Braine, 1985, Experiment 3). This result was taken to indicate poorer left-right than up-down localization. It is possible that poorer discriminability of dashed vertical lines was involved, at least in part.

At this point, attention should be called to two opposing potential artifacts with respect to horizontal versus vertical RT. The narrower vertical stimulus lines that have already been mentioned may have led to slightly longer RTs. However, because of speed-accuracy tradeoff, the higher proportion of errors for the vertical orientation would have been expected to reduce RT on correct trials, compared with RT for the horizontal orientation. Of course, it cannot be demonstrated that these two effects balanced each other exactly. Whatever the net effect, the overall near equality of RT for the two orientations that was obtained in the experiment was the necessary precondition for the crossing interation, which was the basis of the present serendipitous evidence for opposing factors of sensitivity and ambivalence.

We recognize that our conclusions are based on small differences in RT. However, if the hypothesis of opposing factors is correct, large differences would not have been expected in the present experiment. It would be worthwhile to expand the present experiment with both a much easier and a much more difficult discrimination to find whether there would be a clear horizontal advantage for a very difficult discrimination and a clear vertical advantage for a very easy one.

\section{REFERENCES}

FARRELL, W. S., JR. (1979). Coding left and right. Journal of Experimental Psychology: Human Perception \& Performance, 5, 42-51.

Corbaluis, M. C., Beale, I. L. (1983). The ambivalent mind. Chicago: Nelson-Hall.

GoTTSDANKER, R. (1970). A transit-signal methodology for studying reaction time. Behavior Research Methods \& Instrumentation, 2, 6-8.

Greene, S. L., Plastow, E., B Baine, L. G. (1985). Judging the location of features of naturalistic and geometric shapes. Perception \& Psychophysics, 37, 148-154.

HeEley, D. W., \& Timney, B. (1988). Meridional anisotropies of orientation discrimination for sine wave gratings. Vision Research, 28, 337-344.

LeIBowITZ, H. (1953). Some observations and theory on the variation of visual acuity with the orientation of the test-object. Journal of the Optical Society of America, 43, 902-905.

LEIBOWrTZ, H. (1955). Some factors influencing the variability of vernier adjustments. American Journal of Psychology, 68, 266-273.

MCKeE, S. P, \& WestheIMER, G. (1978). Improvement in vernier acuity with practice. Perception \& Psychophysics, 24, 258-262.

Regan, D., \& PrICE, P. (1986). Periodicity in orientation discrimination and the understanding of visual information. Vision Research, 26, 1299-1302.

SANDERS, A. (1980). Stage analysis of reaction processes. In G. Stelmach \& J. Requin (Eds.), Tutorials in motor behavior (pp. 331-354). Amsterdam: North-Holland.

STERNBERG, S. (1969). The discovery of processing stages: Extensions of Donders' method. In W. Koster (Ed.), Attention and performance II (pp. 276-315). Amsterdam: North-Holland.

SUlzer, R. L., \& ZENER, K. A. (1953). A quantitative analysis of relations between stimulus determinants and sensitivity of the visual perception of parallelness. American Psychologist, 8, 444.

(Manuscript received August 26, 1991.) 